\title{
A!
}

This is an electronic reprint of the original article.

This reprint may differ from the original in pagination and typographic detail.

Mercier De Lépinay, Laure; Ockeloen-Korppi, Caspar F.; Malz, Daniel; Sillanpää, Mika A.

\section{Nonreciprocal Transport Based on Cavity Floquet Modes in Optomechanics}

Published in:

Physical Review Letters

DOI:

10.1103/PhysRevLett.125.023603

Published: 10/07/2020

Document Version

Publisher's PDF, also known as Version of record

Please cite the original version:

Mercier De Lépinay, L., Ockeloen-Korppi, C. F., Malz, D., \& Sillanpää, M. A. (2020). Nonreciprocal Transport Based on Cavity Floquet Modes in Optomechanics. Physical Review Letters, 125(2), [023603].

https://doi.org/10.1103/PhysRevLett.125.023603

This material is protected by copyright and other intellectual property rights, and duplication or sale of all or part of any of the repository collections is not permitted, except that material may be duplicated by you for your research use or educational purposes in electronic or print form. You must obtain permission for any other use. Electronic or print copies may not be offered, whether for sale or otherwise to anyone who is not an authorised user. 


\title{
Nonreciprocal Transport Based on Cavity Floquet Modes in Optomechanics
}

\author{
Laure Mercier de Lépinay®, ${ }^{1, *}$ Caspar F. Ockeloen-Korppi®,${ }^{1}$ Daniel Malz®,${ }^{2}$ and Mika A. Sillanpää ${ }^{1}$ \\ ${ }^{1}$ QTF Centre of Excellence, Department of Applied Physics, Aalto University, FI-00076 Aalto, Finland \\ ${ }^{2}$ Max-Planck-Institut für Quantenoptik, Hans-Kopfermann-Strasse 1, D-85748 Garching, Germany
}

(Received 19 December 2019; accepted 15 June 2020; published 10 July 2020)

\begin{abstract}
Directional transport is obtained in various multimode systems by driving multiple, nonreciprocally interfering interactions between individual bosonic modes. However, systems sustaining the required number of modes become physically complex. In our microwave-optomechanical experiment, we show how to configure nonreciprocal transport between frequency components of a single superconducting cavity coupled to two drumhead oscillators. The frequency components are promoted to Floquet modes and generate the missing dimension to realize an isolator and a directional amplifier. A second cavity left free by this arrangement is used to cool the mechanical oscillators and bring the transduction noise close to the quantum limit. We furthermore uncover a new type of instability specific to nonreciprocal coupling. Our approach is generic and can greatly simplify quantum signal processing and the design of topological lattices from low-dimensional systems.
\end{abstract}

DOI: $10.1103 /$ PhysRevLett.125.023603

Introduction.-Lattices with a high degree of complexity are an excellent medium to implement nontrivial topology. While they exist in nature, realizing one with specific functionalities requires a bottom-up synthesis, which remains challenging. Artificial lattices' construction has therefore been investigated in cold atoms [1], photonics [2], superconducting circuits [3-5], and cavity optomechanics $[6,7]$. An intriguing possibility that has received attention recently to demonstrate complex functionalities in a physically low-dimensional system is to complement it with synthetic dimensions [8]. Initially, atoms' internal degrees of freedom were identified as lattice sites [9] aligned along a nonspatial, synthetic dimension supplementing existing spatial dimensions. Floquet quasienergy levels that emerge in a periodically driven nonlinear system [10-16] and multiple or degenerate resonant modes $[12,17,18]$ have also been considered as sites in additional dimensions. Interestingly, nontrivial topology can be designed in synthetic dimensions just as in spatial dimensions, which can lead to nonreciprocal transport [19].

We focus on microwave cavity optomechanics $[20,21]$ where microwave resonators interact with mechanical vibrations. Microwave-optomechanical signal processing, either reciprocal [22-25] or nonreciprocal [26-30], shows some advantages over Josephson-junctions-based processing [31-39]: saturation powers are orders of magnitude higher and superconductivity is not fundamentally necessary. Multimode optomechanical nonreciprocal devices have recently been suggested [26,40-46] and demonstrated [47-52]. Progress in this direction has nonetheless been hindered by the difficulty of fabricating devices with multiple mechanical modes coupled equivalently and strongly to multiple electromagnetic modes. The use of a single cavity mode for several simultaneous operations has been considered for passive detection of stronger processes [53-55]. Kerr-type nonlinearities have also been shown to promote coupling between Floquet modes [56], which are the quasienergy solutions arising via Floquet's theorem offset by multiples of the drive frequency. However, exciting a single cavity mode so that multiple Floquet components actively participate in the dynamics has received little attention, with the notable exception of Ref. [57].

In this Letter, we show that configurable and directional electromagnetic-signal transmission can be obtained in an optomechanical system by designing a loop of interactions in the synthetic plane generated by driven Floquet modes on one hand and multiple mechanical modes on the other hand, to realize a microwave isolator and a directional amplifier. This use of Floquet modes provides a way to simplify nonreciprocal devices and alleviate practical requirements.

Principle.-Let us first consider a multimode cavity optomechanical system as shown in Fig. 1(a), similar to the ones used in a number of directional transduction demonstrations [27-30]. Two mechanical oscillators are both coupled to two cavity modes. Both cavities are excited with two pumping tones. Each set of tones enhances one mechanically mediated coupling mechanism between cavities, and the relative phase of pump tones controls the interference between these two coupling processes.

We demonstrate that this physical system can be recast into the one in Fig. 1(b) where different frequency components from a driven Floquet system of the cavity field play the roles of the two cavity modes. The second cavity is left available for auxiliary optomechanical manipulations. The mediating mechanical modes still participate at their respective resonance frequencies [see Fig. 1(c)], and 


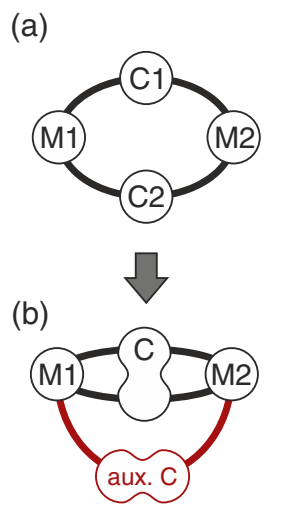

(c)

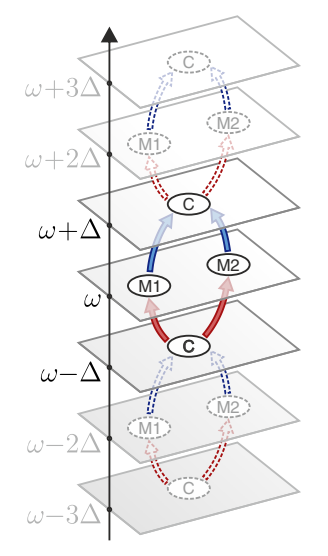

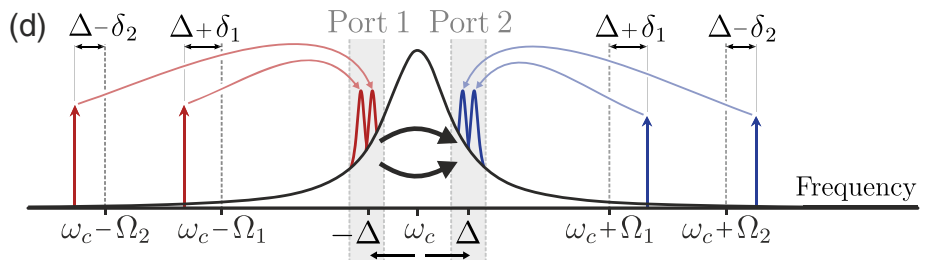

(e)

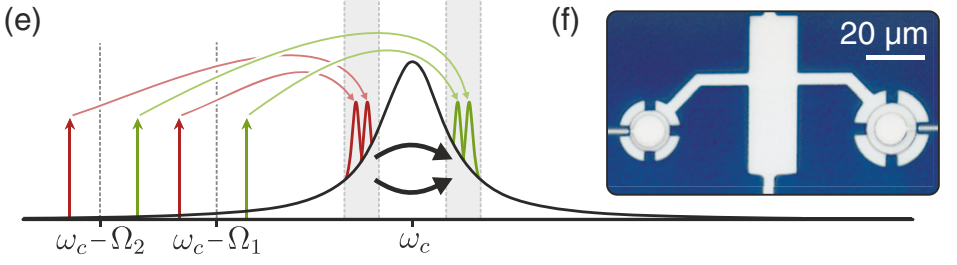

FIG. 1. Principle of Floquet nonreciprocal devices. (a) Scheme of a four-mode nonreciprocal transducer. (b) Simplified system exhibiting nonreciprocal transport between off-resonant components of a single cavity field. An auxiliary cavity mode can be used to sideband cool mechanical modes. (c) Scheme of the double mechanically mediated coupling between Floquet modes represented in the generated synthetic dimensions. Mechanical susceptibilities filtering restrict the coupling picture to the central plaquette. (d) Drive frequencies used to build an optomechanical directional amplifier. The auxiliary cavity pumping scheme is not represented (see Supplemental Material [58]). (e) Similar driving scheme to build an isolator. (f) Optical micrograph of the pair of micromechanical drum oscillators.

their narrow bandwidths play an essential role by restricting the number of Floquet manifolds coupled together.

Let us first consider the configuration of the Floquet directional amplifier. The pertaining pump angular frequencies are as schematized in Fig. 1(d): $\left\{\omega_{c} \pm\left(\Omega_{i}+\delta_{i}+\Delta\right)\right\}_{i=1,2}$. We define $\omega_{c} / 2 \pi$ the frequency of the cavity, $\Omega_{i} / 2 \pi$ of mechanical oscillator $i$, linewidths $\gamma_{i}$ of the oscillators, and the detuning of the pumps $\Delta$ much larger than the mechanical linewidth but smaller than the cavity linewidth $\kappa$. The detunings $\delta_{i}$ are comparable to $\gamma_{i}$ and allow us to drive mechanical susceptibilities out of resonance.

To realize instead a Floquet-mode isolator, all four pump tones are placed close to red sidebands and pumps' angular frequencies become [see Fig. 1(e)] $\left\{\omega_{c}-\left(\Omega_{i}+\delta_{i} \pm \Delta\right)\right\}_{i=1,2}$. In both devices, the pumps drive components of the cavity field $\pm \Delta$ away from resonance. The frequency ranges around these detunings play the roles of the two ports of either device instead of cavity modes [see Fig. 1(d)]. The equations of evolution for electromagnetic and mechanical operators, linearized and with fast rotating terms ignored, display time dependencies that cannot be eliminated by moving to a rotating frame. For example, for the isolator in the frame rotating with $H_{0}=\hbar \omega_{c} a^{\dagger} a+\sum_{i} \hbar\left(\Omega_{i}+\delta_{i}\right) b_{i}^{\dagger} b_{i}$, where $a^{\dagger}, a$ are photonic creation and annihilation operators, and $b_{i}^{\dagger}, b_{i}$ are analogous phononic operators for oscillator $i$, the Langevin equation reads

$$
\begin{aligned}
& \dot{a}=i \sum_{j}\left(G_{j-} e^{i \Delta t}+G_{j+} e^{-i \Delta t}\right) b_{j}-\frac{\kappa}{2} a+s_{\mathrm{in}}, \\
& \dot{b}_{j}=i \delta_{j} b_{j}+i\left(G_{j-}^{*} e^{-i \Delta t}+G_{j+}^{*} e^{i \Delta t}\right) a-\frac{\gamma_{j}}{2} b_{j}+\xi_{j, \text { in }} .
\end{aligned}
$$

$G_{j \pm}=g_{j} \alpha_{j \pm}$ is the enhanced optomechanical coupling for the pump detuned by $\pm \Delta$ associated with mechanical mode $j$, where $g_{j}$ is the single-photon optomechanical coupling of mode $j$ to the cavity and $\alpha_{j \pm}$ is the cavity field amplitude at the frequency of the corresponding pump. The term $s_{\text {in }}=$ $\sqrt{\kappa_{e}} a_{\mathrm{in}}^{e}+\sqrt{\kappa_{i}} a_{\mathrm{in}}^{i}$ describes the signal $\left(a_{\mathrm{in}}^{e}\right)$ and internal noise $\left(a_{\text {in }}^{i}\right)$ of the cavity. The mechanical oscillators' noise input are written as $\xi_{j, \text { in }}=\sqrt{\gamma_{j}} b_{j, \text { in }}$. The total cavity linewidth is the sum of the external and internal loss rates $\kappa=\kappa_{e}+\kappa_{i}$. We introduce cooperativities for each pump $\left\{C_{j \pm}\right\}_{j=1,2}$ and the only relevant phase degree of freedom $\varphi$ [26] between pumps, such that $G_{1-}=\sqrt{\gamma_{1} \kappa C_{1-} / 4} e^{+i \varphi / 2}, G_{2-}=$ $\sqrt{\gamma_{2} \kappa C_{2-} / 4} e^{-i \varphi / 2}$, and $G_{j+}=\sqrt{\gamma_{j} \kappa C_{j+} / 4}$ for $j=1,2$. The phase $\varphi$ is a crucial parameter as it determines the nonreciprocal nature of the coupling and therefore the directionality of the transduction. Since $\gamma_{j}$ is by far the smallest frequency scale of the system, narrow mechanical susceptibilities restrict the number of relevant harmonics to two only at detunings $-\Delta$ and $+\Delta$ from the cavity resonance frequency (see Supplemental Material [58]). These Floquet components define the two ports of the device, hereafter named, respectively, ports 1 and 2.

Eliminating phononic operators from Fourier transformed Eq. (1), it follows that a two-vector of cavity operators is invariant under the evolution equations: $A(\omega)=$ $[a(\omega-\Delta) a(\omega+\Delta)]^{T}$ in the case of the isolator and $A(\omega)=\left[a(\omega-\Delta) a^{\dagger}(\omega-\Delta)\right]^{T}$ in the case of the amplifier. Defining a global cavity susceptibility $2 \times 2$ matrix $\chi(\omega)$ [58], the vector $A$ is related to similarly defined drive vectors $A_{\text {in }}^{e}$ and $A_{\text {in }}^{i}$ by $A(\omega)=\chi(\omega)\left[\sqrt{\kappa_{e}} A_{\text {in }}^{e}(\omega)+\sqrt{\kappa_{i}} A_{\text {in }}^{i}(\omega)\right]$. Using an analogous definition for the cavity output rate $A_{\text {out }}$, the input-output relation reads $A_{\text {out }}=A_{\text {in }}^{e}-\sqrt{\kappa_{e}} A$. Therefore, the scattering matrix $S$ defined by $A_{\text {out }}=S A_{\text {in }}^{e}$ (temporarily omitting noise terms) is $S(\omega)=\mathbb{I}_{2}-\kappa_{e} \chi(\omega)$. 

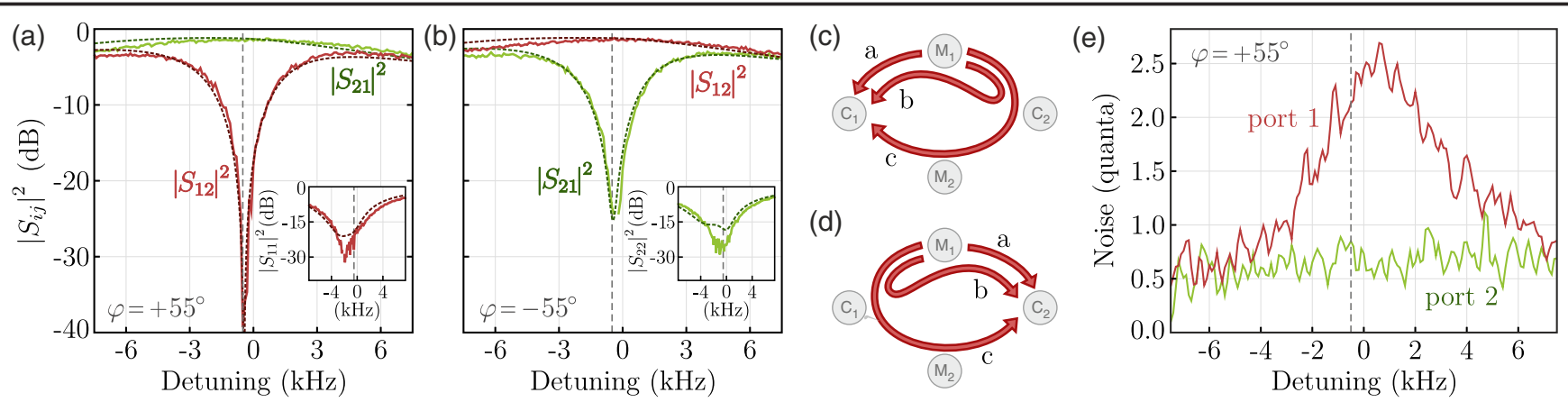

FIG. 2. Microwave isolator. (a) Scattering parameters $S_{12}$ (solid red line) and $S_{21}$ (light green solid line) for $\varphi=55^{\circ}$ and fits (darker dashed lines). The dashed gray line marks the optimal working frequency. (Inset) Reflection $S_{11}$ on isolated port 1 and fit (darker dashed line). (b) Same as (a) for $\varphi=-55^{\circ}$. (Inset) Reflection $S_{22}$ on isolated port and fit (darker dashed line). (c) Schematic of the paths taken by noise from mechanical oscillator 1 to port 1. (d) Same as (c) for noise from mechanical oscillator 1 to port 2. (e) For $\varphi=+55^{\circ}$, noise at port 1 (solid red) corresponding to backward-propagating noise for the isolator with this phase and at port 2 (solid light green) corresponding to forward-propagating noise.

The elements $S_{i j}$ of this matrix define the scattering parameters between ports $j$ and $i$.

Experiment.-An on-chip microwave $L C$ circuit lithographied in aluminum sustains two electromagnetic cavity modes that we coin "primary" and "auxiliary." The former is used to establish nonreciprocal transfer and the latter to sideband cool the mechanical modes [60] in order to reduce noise and broaden the bandwidth. The cavity modes have respective frequencies $\omega_{c} / 2 \pi=4.98$ and $\omega_{c}^{a} / 2 \pi=6.62 \mathrm{GHz}$ and internal and external decay rates $\kappa_{e} / 2 \pi=1.3 \mathrm{MHz}$, $\kappa_{i} / 2 \pi=190 \mathrm{kHz}$, and $\kappa_{e}^{a} / 2 \pi=900, \kappa_{i}^{a} / 2 \pi=580 \mathrm{kHz}$. The circuit includes two vacuum-gap capacitors [see Fig. 1(f)] whose top plate is allowed to move freely, materializing mechanical oscillators of frequencies $\Omega_{1} / 2 \pi=6.69$ and $\Omega_{2} / 2 \pi=9.03 \mathrm{MHz}$ and intrinsic decay rates $\gamma_{1}^{0} / 2 \pi=55$ and $\gamma_{2}^{0} / 2 \pi=110 \mathrm{~Hz}$. The chip is mounted in a dilution cryostat to be operated at a temperature of $10 \mathrm{mK}$.

Isolator.-We now discuss the configuration of Fig. 1(e) that employs only red-sideband tones. The global susceptibility matrix in the basis defined by $A$ is modified by a coupling matrix $T$,

$\chi^{-1}(\omega)=\left(\begin{array}{cc}\frac{\kappa}{2}-i(\omega-\Delta) & 0 \\ 0 & \frac{\kappa}{2}-i(\omega+\Delta)\end{array}\right)+\left(\begin{array}{ll}T_{11} & T_{12} \\ T_{21} & T_{22}\end{array}\right)$.

Diagonal coupling terms $T_{11}\left(T_{22}\right)$ account for standard backaction of mechanical modes on Floquet cavity modes from the two pumps detuned by $-\Delta(+\Delta)$. Off-diagonal terms $T_{i j}(i \neq j)$ also involve one contribution from each mechanically mediated coupling path between Floquet cavity modes,

$$
\begin{aligned}
& T_{12}(\omega)=G_{1-} G_{1+} \chi_{m, 1}(\omega)+G_{2-} G_{2+} \chi_{m, 2}(\omega), \\
& T_{21}(\omega)=G_{1-}^{*} G_{1+}^{*} \chi_{m, 1}(\omega)+G_{2-}^{*} G_{2+}^{*} \chi_{m, 2}(\omega),
\end{aligned}
$$

where $\chi_{m, j}$ is the susceptibility of mechanical mode $j$, centered on $-\delta_{j}: \chi_{m, j}(\omega)=\left[\gamma_{j} / 2-i\left(\omega+\delta_{j}\right)\right]^{-1}$. Offdiagonal elements of the scattering matrix $S_{12}$ and $S_{21}$ are proportional to $T_{12}$ and $T_{21}$, respectively (see Supplemental Material [58]). Therefore, to obtain, e.g., isolation of port 1 $\left(S_{12}=0\right)$, it suffices to cancel out $T_{12}$. In order to maintain simultaneous transfer in the other direction, one must ensure that $S_{21}$, and therefore $T_{21}$, is concurrently as high as possible. This asymmetry is made possible thanks to the phase shift of each coupling path provided by the offresonance participation of either mechanical oscillator [58].

The experiment is prepared by sideband cooling mechanical oscillators through the auxiliary cavity down to $n_{\text {leff }} \simeq 4.0$ and $n_{\text {2eff }} \simeq 8.9$ quanta. Corresponding effective mechanical linewidths are $\gamma_{1} / 2 \pi \simeq 1.7$ and $\gamma_{2} / 2 \pi \simeq 1.9 \mathrm{kHz}$. The primary cavity is pumped with detuning $\Delta / 2 \pi=30 \mathrm{kHz}$, much larger than the effective mechanical damping rates, with cooperativities $\left\{C_{1-}, C_{1+}\right.$, $\left.C_{2-}, C_{2+}\right\}=\{3.9,2.5,3.7,2.4\}$ and additional detunings $\delta_{1} / 2 \pi=-\delta_{2} / 2 \pi=1 \mathrm{kHz}$. By measuring the scattering of a weak probe tone sent along with the pumps [58], we show in Fig. 2(a) an optimal isolation for $\varphi=+55^{\circ}$ of $\left|S_{12}\right|^{2} \simeq$ $-39.3 \mathrm{~dB}$ with $\left|S_{21}\right|^{2} \simeq-1.3 \mathrm{~dB}$ insertion loss. We also demonstrate for the opposite phase $\varphi=-55^{\circ}$ a device working in the reversed direction in Fig. 2(b) with $\left|S_{21}\right|^{2} \simeq$ $-24.5 \mathrm{~dB}$ isolation and $\left|S_{12}\right|^{2} \simeq-1.2 \mathrm{~dB}$ insertion loss. The bandwidth around $3 \mathrm{kHz}$ is comparable to the effective mechanical linewidths. Because of relatively small mechanical frequency separation, pumps also excite the mechanical mode they are not intended to drive, leading to dynamical backaction taken into account in the theoretical fits presented throughout the Letter.

The noise in the device arises mainly from mechanical thermal noise [26] which propagates through three paths as indicated in Fig. 2(c): path "a" is the direct conversion of phonons into the same amount of cavity photons. The two others ("b" and "c") follow the same route as signals across 

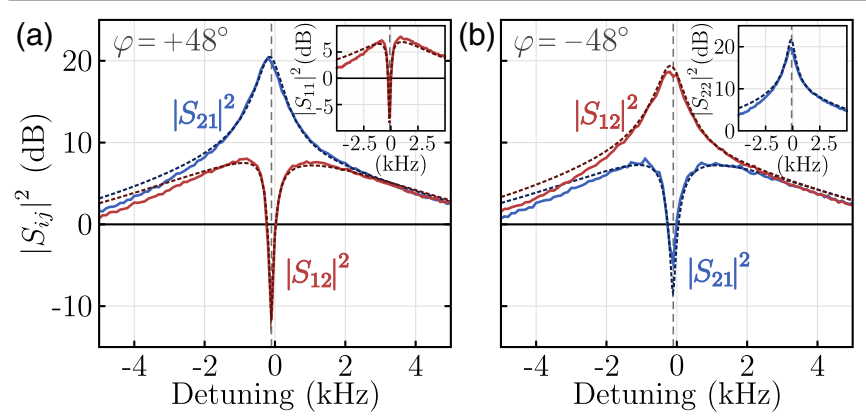

FIG. 3. Directional amplification. (a) Scattering parameters $S_{12}$ (solid red line) and $S_{21}$ (solid blue line) for $\varphi=+48^{\circ}$ and fits with expressions from the text (darker dashed lines). (Inset) Reflection $S_{11}$ (solid line) on isolated port and fit (darker dashed line). (b) Same as (a) for the opposite phase $\varphi=-48^{\circ}$. (Inset) Reflection $S_{22}$ on isolated port (solid line) and fit (darker dashed line).

the device and interfere destructively at the isolated port. Therefore, only path a contributes to backward-propagating noise, which is thus simply half of the mechanical oscillators' total occupation: $n_{\text {back }}=\frac{1}{2}\left(n_{1}+n_{2}+1\right)$ in the limit of high cooperativities and ideal cavity. Here we maintain this noise around $n_{\text {back }} \simeq 2.5$ quanta [see Fig. 2(e)]. By comparison, without sideband cooling, the expected inputport noise is 35 photons in the ideal case. At the other port [see Fig. 2(d)], direct path a interferes somewhat destructively with the sum of indirect paths $b$ and $c$ and mitigates total fluctuations, which results in very small output noise of thermal origin, and thus $n_{\text {out }} \simeq 0.6$ quanta [see Fig. 2(e)].

Directional amplifier.-OWing to blue-sideband driving, the $S$ parameters of the amplifier relate $a(\omega-\Delta)$ to $a^{\dagger}(\omega-\Delta)$ : they exchange quadratures between input and output ports. This translates the phase-preserving but phase-conjugating nature of the device [61] and entails that signals sent at a frequency $\omega_{c}-\Delta-\nu$ are converted at $\omega_{c}+\Delta+\nu$ [30]; that is, the output frequency is mirrored around the port central frequency. As long as the detunings $\Delta$ are small compared to $\kappa$, the gain $G$ in the limit of ideal cavities and large cooperativities remains the same as for separate-cavity amplifiers $[26,30,58]$,

$$
G=\frac{4 C_{1-} C_{1+}}{\left(C_{1-}-C_{1+}\right)^{2}}
$$

With similar mechanical precooling $\left(n_{1} \simeq 2.9, n_{2} \simeq 8.1\right.$, $\left.\gamma_{1} / 2 \pi \simeq 1.6 \mathrm{kHz}, \gamma_{2} / 2 \pi \simeq 0.9 \mathrm{kHz}\right)$ and same detuning $\Delta$ as for the isolator, we use cooperativities $\left\{C_{1-}, C_{1+}\right.$, $\left.C_{2-}, C_{2+}\right\} \simeq\{4.2,3.2,5.3,2.6\}$ to demonstrate in Fig. 3(a) for $\varphi=+48^{\circ}$ a maximum amplification gain of $G \simeq$ $\left|S_{21}\right|^{2} \simeq 20.3 \mathrm{~dB}$ and a simultaneous isolation of $\left|S_{12}\right|^{2} \simeq$ $-11.7 \mathrm{~dB}$. The amplification and the isolation bandwidths 1.5 and $1 \mathrm{kHz}$, respectively, are again comparable to the mechanical linewidths, but lower than those of the isolator since they are not enhanced by parasitic coupling (see Supplemental Material [58]). Figure 3(b) also shows a gain

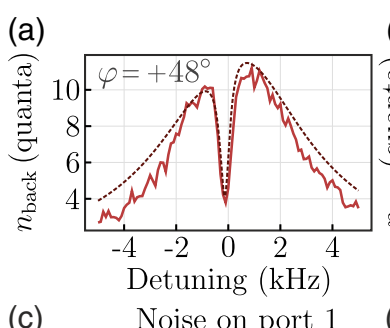

(b)
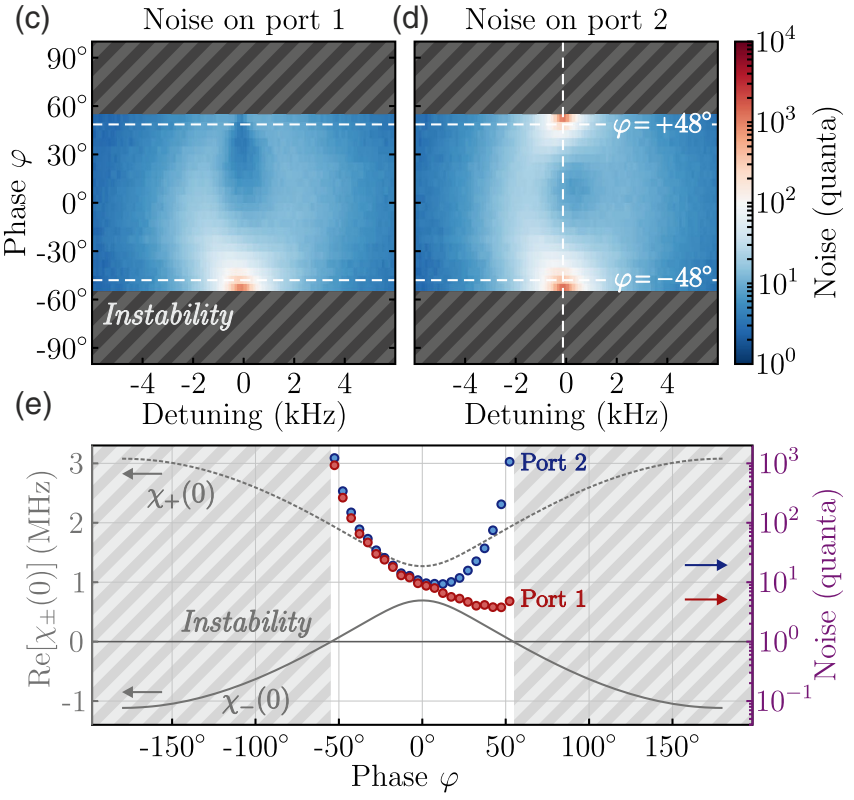

FIG. 4. Noise and phase-dependent instability. (a) Backwardpropagating noise for optimal phase $\varphi=+48^{\circ}$, corresponding to noise at port 1 (solid red) and theory superimposed (darker dashed line). (b) Added noise for optimal phase $\varphi=+48^{\circ}$ (solid blue) and theory (darker dashed line). (c) Noise at port 1 as a function of frequency and pump phase. Gray dashed area: unstable region. White horizontal dashed lines: optimal phases $\varphi= \pm 48^{\circ}$. (d) Same as (c) but at port 2. Dashed vertical white line: frequency of optimal directionality. (e) Calculated real parts of the optical eigensusceptibilities at zero frequency (solid and dashed gray lines, right-side scale). The lower real part is negative for $|\varphi|>55^{\circ}$, causing the instability. Noise at optimal frequency [cut of (d) along dashed vertical line] at port 2 (blue dots, left-side scale) diverges at the onset of the instability, as does noise at port 1 (red dots, left-side scale) although noise paths destructive interference at $\varphi=+48^{\circ}$ limits the maximum measured noise.

of $\left|S_{12}\right|^{2} \simeq 18.5 \mathrm{~dB}$ and a simultaneous isolation of $\left|S_{21}\right|^{2} \simeq$ $-4.7 \mathrm{~dB}$ with the opposite phase $\varphi=-48^{\circ}$. However, contrary to the case of the isolator, only one port can be impedance matched to the transmission line due to the asymmetric pumping of red sidebands of one Floquet mode and blue sidebands of the other [58]. As a result of this asymmetry, regardless of the phase, port 1 displays low reflectivity $\left|S_{11}\right|^{2}$ and port 2 a large one $\left|S_{22}\right|^{2}$ [see insets to Figs. 3(a) and 3(b)]. The optimal configuration is therefore $\varphi=+48^{\circ}$, which suppresses power reflected on the input port by $\left|S_{11}\right|^{2} \simeq-7.4 \mathrm{~dB}$.

In contrast to the isolator, fluctuations propagating across the amplifier are amplified, which results in a 
generally high noise. However, we measure in this configuration only $n_{\text {back }} \simeq 3.8$ quanta at port 1 when it is isolated at $\varphi=+48^{\circ}$ [see Fig. 4(c)]. Indeed, as for the isolator, amplified paths $\mathrm{b}$ and $\mathrm{c}$ interfere destructively and only the nonamplified noise from direct path a contributes to the backward noise, which is at high cooperativities half the total mechanical occupancy and is reduced by active cooling. Output noise results from the same three-path interference as in the isolator and at phase $\varphi=+48^{\circ}$ the added noise is $n_{\text {add }}=n_{\text {out }} / G \simeq 2.1$ photons [see Fig. 4(b)], close to the quantum limit of 0.5 photons. On the other hand, for $\varphi=-48^{\circ}$, port 2 , which displays high reflective gain $S_{22}$, outputs a backward-propagating noise reaching $n_{\text {back }} \simeq 360$ quanta even with aggressive auxiliary cooling. This asymmetry between noise at phases $\varphi= \pm 48^{\circ}$ is visible in Figs. 4(c) and 4(d). This represents a second reason, together with asymmetric impedance matching, for which the behavior of the device is not inverted by simply changing the sign of the phase.

Phase-dependent instability.-Nonreciprocal amplification furthermore reveals a new instability for a range of phases. Contrary to optomechanical instability, it does not arise directly from a strong blue-sideband driving but is related to the emergence of an unstable eigenmode of the susceptibility $\chi$. Figures 4(c) and 4(d) show the amplifier noise for a limited range of phase parameters $|\varphi|<55^{\circ}$ because the device is unstable for the rest of the $360^{\circ}$ range. As shown in Fig. 4(e), the onset of the instability coincides with the phase at which one of the eigenvalues of the electromagnetic susceptibility matrix acquires a negative real part. Figure 4(e) furthermore shows that noise diverges at these phases. In the isolator case, the eigenvalue with lower real part is stabilized by dynamical backaction and this real-part never crosses zero. The observed instability is therefore specific to nonreciprocally coupled, nonstabilized multimode devices and relates to the instability observed in other phase-preserving nonreciprocal coupling situations [62].

Conclusion.-We have theoretically and experimentally demonstrated a new archetype of nonreciprocal optomechanical devices based on the interference of Floquet modes in a single cavity. This physical simplification allows us to accommodate auxiliary optomechanical manipulations of mechanical oscillators to closely approach the quantum limit of the transduction. We foresee that this approach can greatly simplify signal processing in other physical platforms involving resonators. Finally, we uncovered a class of instability arising in nonreciprocally coupled systems provided they are not stabilized by dynamical backaction.

We thank Andreas Nunnenkamp, Clara Wanjura, and Matteo Brunelli for useful discussions. This work was supported by the Academy of Finland (Contracts No. 308290 and No. 307757), by the European Research Council (615755-CAVITYQPD), and by the Aalto Centre for Quantum Engineering. The work was performed as part of the Academy of Finland Centre of Excellence program (Project No. 312057). We acknowledge funding from the European Union's Horizon 2020 research and innovation program under Grant Agreement No. 732894 (FETPRO HOT). D. M. acknowledges funding from ERC Advanced Grant QENOCOBA under the EU Horizon 2020 program (Grant Agreement No. 742102). We acknowledge the facilities and technical support of Otaniemi research infrastructure for Micro and Nanotechnologies (OtaNano) that is part of the European Microkelvin Platform.

*Corresponding author.

laure.mercierdelepinay@aalto.fi

[1] N. Goldman, J. C. Budich, and P. Zoller, Topological quantum matter with ultracold gases in optical lattices, Nat. Phys. 12, 639 (2016).

[2] L. Lu, J. D. Joannopoulos, and M. Soljačić, Topological photonics, Nat. Photonics 8, 821 (2014).

[3] J. Koch, A. A. Houck, K. Le Hur, and S. M. Girvin, Timereversal-symmetry breaking in circuit-QED-based photon lattices, Phys. Rev. A 82, 043811 (2010).

[4] D. I. Tsomokos, S. Ashhab, and F. Nori, Using superconducting qubit circuits to engineer exotic lattice systems, Phys. Rev. A 82, 052311 (2010).

[5] A. J. Kollár, M. Fitzpatrick, and A. A. Houck, Hyperbolic lattices in circuit quantum electrodynamics, Nature (London) 571, 45 (2019).

[6] V. Peano, C. Brendel, M. Schmidt, and F. Marquardt, Topological Phases of Sound and Light, Phys. Rev. X 5, 031011 (2015).

[7] M. Schmidt, S. Kessler, V. Peano, O. Painter, and F. Marquardt, Optomechanical creation of magnetic fields for photons on a lattice, Optica 2, 635 (2015).

[8] T. Ozawa and H. M. Price, Topological quantum matter in synthetic dimensions, Nat. Rev. Phys. 1, 349 (2019).

[9] A. Celi, P. Massignan, J. Ruseckas, N. Goldman, I. B. Spielman, G. Juzeliūnas, and M. Lewenstein, Synthetic Gauge Fields in Synthetic Dimensions, Phys. Rev. Lett. 112, 043001 (2014).

[10] M. C. Rechtsman, J. M. Zeuner, Y. Plotnik, Y. Lumer, D. Podolsky, F. Dreisow, S. Nolte, M. Segev, and A. Szameit, Photonic Floquet topological insulators, Nature (London) 496, 196 (2013).

[11] I. Martin, G. Refael, and B. Halperin, Topological Frequency Conversion in Strongly Driven Quantum Systems, Phys. Rev. X 7, 041008 (2017).

[12] Q. Lin, X.-Q. Sun, M. Xiao, Sh.-Ch. Zhang, and Sh. Fan, A three-dimensional photonic topological insulator using a two-dimensional ring resonator lattice with a synthetic frequency dimension, Sci. Adv. 4, eaat2774 (2018).

[13] Y. Baum and G. Refael, Setting Boundaries with Memory: Generation of Topological Boundary States in FloquetInduced Synthetic Crystals, Phys. Rev. Lett. 120, 106402 (2018).

[14] P. J. D. Crowley, I. Martin, and A. Chandran, Topological classification of quasiperiodically driven quantum systems, Phys. Rev. B 99, 064306 (2019). 
[15] C. W. Peterson, W. A. Benalcazar, M. Lin, T. L. Hughes, and G. Bahl, Strong Nonreciprocity in Modulated Resonator Chains through Synthetic Electric and Magnetic Fields, Phys. Rev. Lett. 123, 063901 (2019).

[16] A. Dutt, M. Minkov, Q. Lin, L. Yuan, D. A. B. Miller, and S. Fan, Experimental band structure spectroscopy along a synthetic dimension, Nat. Commun. 10, 3122 (2019).

[17] T. Andrijauskas, I. B. Spielman, and G. Juzeliūnas, Topological lattice using multi-frequency radiation, New J. Phys. 20, 055001 (2018).

[18] Y. Chen, Y.-L. Zhang, Zh. Shen, Ch.-L. Zou, G.-C. Guo, and Ch.-H. Dong, Synthetic gauge field in a single optomechanical resonator, arXiv:1908.04456.

[19] C. C. Wanjura, M. Brunelli, and A. Nunnenkamp, Topological framework for directional amplification in drivendissipative cavity arrays, Nat. Commun. 11, 3149 (2020).

[20] C. A. Regal, J. D. Teufel, and K. W. Lehnert, Measuring nanomechanical motion with a microwave cavity interferometer, Nat. Phys. 4, 555 (2008).

[21] J. D. Teufel, T. Donner, D. Li, J. W. Harlow, M. S. Allman, K. Cicak, A. J. Sirois, J. D. Whittaker, K. W. Lehnert, and R. W. Simmonds, Sideband cooling of micromechanical motion to the quantum ground state, Nature (London) 475, 359 (2011).

[22] F. Massel, T. T. Heikkilä, J.-M. Pirkkalainen, S. U. Cho, H. Saloniemi, P. J. Hakonen, and M. A. Sillanpää, Microwave amplification with nanomechanical resonators, Nature (London) 480, 351 (2011).

[23] C. F. Ockeloen-Korppi, E. Damskägg, J.-M. Pirkkalainen, T. T. Heikkilä, F. Massel, and M. A. Sillanpää, Low-Noise Amplification and Frequency Conversion with a Multiport Microwave Optomechanical Device, Phys. Rev. X 6, 041024 (2016).

[24] C. F. Ockeloen-Korppi, E. Damskägg, J.-M. Pirkkalainen, T. T. Heikkilä, F. Massel, and M. A. Sillanpää, Noiseless Quantum Measurement and Squeezing of Microwave Fields Utilizing Mechanical Vibrations, Phys. Rev. Lett. 118, 103601 (2017).

[25] L. D. Tóth, N. R. Bernier, A. Nunnenkamp, A. K. Feofanov, and T. J. Kippenberg, A dissipative quantum reservoir for microwave light using a mechanical oscillator, Nat. Phys. 13, 787 (2017).

[26] D. Malz, L. D. Tóth, N. R. Bernier, A. K. Feofanov, T. J. Kippenberg, and A. Nunnenkamp, Quantum-Limited Directional Amplifiers with Optomechanics, Phys. Rev. Lett. 120, 023601 (2018).

[27] G. A. Peterson, F. Lecocq, K. Cicak, R. W. Simmonds, J. Aumentado, and J.D. Teufel, Demonstration of Efficient Nonreciprocity in a Microwave Optomechanical Circuit, Phys. Rev. X 7, 031001 (2017).

[28] N. R. Bernier, L. D. Tóth, A. Koottandavida, M. A. Ioannou, D. Malz, A. Nunnenkamp, A. K. Feofanov, and T. J. Kippenberg, Nonreciprocal reconfigurable microwave optomechanical circuit, Nat. Commun. 8, 604 (2017).

[29] S. Barzanjeh, M. Wulf, M. Peruzzo, M. Kalaee, P. B. Dieterle, O. Painter, and J.M. Fink, Mechanical on-chip microwave circulator, Nat. Commun. 8, 953 (2017).

[30] L. Mercier de Lépinay, E. Damskägg, C. F. OckeloenKorppi, and M. A. Sillanpää, Realization of Directional
Amplification in a Microwave Optomechanical Device, Phys. Rev. Applied 11, 034027 (2019).

[31] R. Movshovich, B. Yurke, P. G. Kaminsky, A. D. Smith, A. H. Silver, R. W. Simon, and M. V. Schneider, Observation of Zero-Point Noise Squeezing Via a JosephsonParametric Amplifier, Phys. Rev. Lett. 65, 1419 (1990).

[32] M. A. Castellanos-Beltran, K. D. Irwin, G. C. Hilton, L. R. Vale, and K. W. Lehnert, Amplification and squeezing of quantum noise with a tunable Josephson metamaterial, Nat. Phys. 4, 929 (2008).

[33] B. Abdo, K. Sliwa, L. Frunzio, and M. Devoret, Directional Amplification with a Josephson Circuit, Phys. Rev. X 3, 031001 (2013).

[34] L. Zhong, E. P. Menzel, R. Di Candia, P. Eder, M. Ihmig, A. Baust, M. Haeberlein, E. Hoffmann, K. Inomata, T. Yamamoto, Y. Nakamura, E. Solano, F. Deppe, A. Marx, and R. Gross, Squeezing with a flux-driven Josephson parametric amplifier, New J. Phys. 15, 125013 (2013).

[35] B. Abdo, K. Sliwa, S. Shankar, M. Hatridge, L. Frunzio, R. Schoelkopf, and M. Devoret, Josephson Directional Amplifier for Quantum Measurement of Superconducting Circuits, Phys. Rev. Lett. 112, 167701 (2014).

[36] C. Eichler, Y. Salathe, J. Mlynek, S. Schmidt, and A. Wallraff, Quantum-Limited Amplification and Entanglement in Coupled Nonlinear Resonators, Phys. Rev. Lett. 113, 110502 (2014).

[37] K. M. Sliwa, M. Hatridge, A. Narla, S. Shankar, L. Frunzio, R. J. Schoelkopf, and M. H. Devoret, Reconfigurable Josephson Circulator/Directional Amplifier, Phys. Rev. X 5, 041020 (2015).

[38] F. Lecocq, L. Ranzani, G. A. Peterson, K. Cicak, R. W. Simmonds, J. D. Teufel, and J. Aumentado, Nonreciprocal Microwave Signal Processing with a Field-Programmable Josephson Amplifier, Phys. Rev. Applied 7, 024028 (2017).

[39] M. P. Westig and T. M. Klapwijk, Josephson Parametric Reflection Amplifier with Integrated Directionality, Phys. Rev. Applied 9, 064010 (2018).

[40] M. Hafezi and P. Rabl, Optomechanically induced nonreciprocity in microring resonators, Opt. Express 20, 7672 (2012).

[41] A. Metelmann and A. A. Clerk, Nonreciprocal Photon Transmission and Amplification via Reservoir Engineering, Phys. Rev. X 5, 021025 (2015).

[42] X.-W. Xu and Y. Li, Optical nonreciprocity and optomechanical circulator in three-mode optomechanical systems, Phys. Rev. A 91, 053854 (2015).

[43] X.-W. Xu, Y. Li, A.-X. Chen, and Y.-X. Liu, Nonreciprocal conversion between microwave and optical photons in electro-optomechanical systems, Phys. Rev. A 93, 023827 (2016).

[44] Y. Li, Y. Y. Huang, X. Z. Zhang, and L. Tian, Optical directional amplification in a three-mode optomechanical system, Opt. Express 25, 18907 (2017).

[45] C. Jiang, L. N. Song, and Y. Li, Directional amplifier in an optomechanical system with optical gain, Phys. Rev. A 97, 053812 (2018).

[46] Y. Jiang, S. Maayani, T. Carmon, F. Nori, and H. Jing, Nonreciprocal Phonon Laser, Phys. Rev. Applied 10, 064037 (2018). 
[47] S. Hua, J. Wen, X. Jiang, Q. Hua, L. Jiang, and M. Xiao, Demonstration of a chip-based optical isolator with parametric amplification, Nat. Commun. 7, 13657 (2016).

[48] Z. Shen, Y.-L. Zhang, Y. Chen, C.-L. Zou, Y.-F. Xiao, X.-B. Zou, F.-W. Sun, G.-C. Guo, and C.-H. Dong, Experimental realization of optomechanically induced non-reciprocity, Nat. Photonics 10, 657 (2016).

[49] F. Ruesink, M.-A. Miri, A. Alù, and E. Verhagen, Nonreciprocity and magnetic-free isolation based on optomechanical interactions, Nat. Commun. 7, 13662 (2016).

[50] K. Fang, J. Luo, A. Metelmann, M. H. Matheny, F. Marquardt, A. A. Clerk, and O. Painter, Generalized non-reciprocity in an optomechanical circuit via synthetic magnetism and reservoir engineering, Nat. Phys. 13, 465 (2017).

[51] Z. Shen, Y.-L. Zhang, Y. Chen, F.-W. Sun, X.-B. Zou, G.-C. Guo, C.-L. Zou, and C.-H. Dong, Reconfigurable optomechanical circulator and directional amplifier, Nat. Commun. 9, 1797 (2018).

[52] F. Ruesink, J. P. Mathew, M.-A. Miri, A. Alù, and E. Verhagen, Optical circulation in a multimode optomechanical resonator, Nat. Commun. 9, 1798 (2018).

[53] F. Lecocq, J. B. Clark, R. W. Simmonds, J. Aumentado, and J. D. Teufel, Quantum Nondemolition Measurement of a Nonclassical State of a Massive Object, Phys. Rev. X 5, 041037 (2015).

[54] E. E. Wollman, C. U. Lei, A. J. Weinstein, J. Suh, A. Kronwald, F. Marquardt, A. A. Clerk, and K. C. Schwab, Quantum squeezing of motion in a mechanical resonator, Science 349, 952 (2015).
[55] C. F. Ockeloen-Korppi, E. Damskägg, J. M. Pirkkalainen, M. Asjad, A. A. Clerk, F. Massel, M. J. Woolley, and M. A. Sillanpää, Stabilized entanglement of massive mechanical oscillators, Nature (London) 556, 478 (2018).

[56] L. Qiu, I. Shomroni, M. A. Ioannou, N. Piro, D. Malz, A. Nunnenkamp, and T. J. Kippenberg, Floquet dynamics in the quantum measurement of mechanical motion, Phys. Rev. A 100, 053852 (2019).

[57] H. Xu, L. Jiang, A. A. Clerk, and J. G. E. Harris, Nonreciprocal control and cooling of phonon modes in an optomechanical system, Nature (London) 568, 65 (2019).

[58] See Supplemental Material at http://link.aps.org/ supplemental/10.1103/PhysRevLett.125.023603 for experimental and theoretical details, which includes Ref. [59].

[59] Z. Gong, Y. Ashida, K. Kawabata, K. Takasan, S. Higashikawa, and M. Ueda, Topological Phases of NonHermitian Systems, Phys. Rev. X 8, 031079 (2018).

[60] A. Schliesser, R. Rivière, G. Anetsberger, O. Arcizet, and T. J. Kippenberg, Resolved-sideband cooling of a micromechanical oscillator, Nat. Phys. 4, 415 (2008).

[61] C. M. Caves, Quantum limits on noise in linear amplifiers, Phys. Rev. D 26, 1817 (1982).

[62] A. Gloppe, P. Verlot, E. Dupont-Ferrier, A. G. Kuhn, A. Siria, P. Poncharal, G. Bachelier, P. Vincent, and O. Arcizet, Bidimensional nano-optomechanics and topological backaction in a non-conservative radiation force field, Nat. Nanotechnol. 9, 920 (2014). 